\title{
Primary Melanoma of the Bladder at Puerperium: Case Report
}

\section{Rubio Galisteo $\mathrm{JM}^{1 *}$, Gomez Gomez $\mathrm{E}^{1}$, Valero Rosa $\mathrm{J}^{1}$, Salguero Segura $\mathrm{J}^{1}$, Pineda Reyes $\mathrm{B}^{2}$, Gonzalez $T^{3}$, Barbudo Merino $J^{4}$, Ruiz Garcia $\mathrm{JM}^{1}$ and Requena Tapia $\mathrm{MJ}^{1}$}

${ }^{1}$ Urology Department, Hospital Universitario Reina Sofia, Spain

${ }^{2}$ Ginecology Department, Hospital Universitario Reina Sofia, Spain

${ }^{3}$ Pathological Anatomy Department, Hospital Universitario Reina Sofia, Spain

${ }^{4}$ Emergency Department, Hospital Universitario Reina Sofia, Spain

*Corresponding author: Rubio Galisteo JM, Urology Department, Hospital Universitario Reina Sofía, Av Menendez Pidal S/N, UGC Urología, Córdoba, CP: 14004, Spain, Tel: +3460-004-5566

\begin{abstract}
Primary malignant melanoma of the urinary bladder is a sporadic disease and very little described in the literature. A 39-years-old female at the end of her pregnancy without previous history of skin disease was presented with hematuria after cesarean and with constitutional syndrome. After the study, the patient was diagnosed with metastatic bladder melanoma. Other locations of primary injury were ruled out. The patient died a month and a half after the diagnosis.
\end{abstract}

\section{Abbreviations}

CT Scan: Computed Tomography Scan; TUR: Transurethral Resection

\section{Introduction}

Malignant melanoma of urinary bladder is a sporadic disease, very little described in the literature. Approximately only about 50 cases have been reported throughout history. This histological type is part of the group of tumours of mesenchymal origin. These tumours are accounting for 0.2 to $5 \%$ of all bladder tumours. The bladder localization is an uncommon region for melanoma, being only $0.2 \%$ of all melanomas. It belongs to the mucosal melanomas; these tumours generally carry a worse prognosis than those arising from cutaneous sites. In general, patients diagnosed with mucosal melanomas are older, with a median age of 70 years [1-6].

We present a case of primary malignant melanoma of urinary bladder in a 39-years-old postpartum woman.

\section{Case Presentation}

A healthy 39-years-old female is presented at the end of her first pregnancy. The patient gives a history of $10 \mathrm{~kg}$ lost at the last months, and urinary tract infection treated with antibiotics with a urine culture positive to $E$. coli one month earlier. The patient is admitted to the hospital with contractions in context clinic to birthing. At that moment, the patient present general discomfort, dyspnea, tachycardia and sweating. Some hours later, it's necessary to practice an urgent caesarean for loss of fetal wellbeing with the necessity of urinary catheterization showing gross hematuria. After caesarean and the persistence of dyspnea, a chest X-ray is taken showing cottony infiltrates, so a computed tomography scan is taken viewing an excrescence lesion in bladder suggestive of bladder cancer with multiple metastatic lesions in the liver, spleen, left adrenal, presacral region, peritoneum and the retroperitoneal region as well as in axial skeleton and pulmonary parenchyma (Figure 1).

At the moment of birth, the child was healthy with an APGAR test of $7 / 10$, and no other disease expression appeared after the cesarean.

To the findings in CT scan, it is decided in committee realization of transurethral resection (TUR) and bi-

Citation: Rubio GJM, Gomez GE, Valero RJ, Salguero SJ, Pineda RB, et al. (2020) Primary Melanoma of the Bladder at Puerperium: Case Report. Int Arch Urol Complic 6:073. doi.org/10.23937/24695742/1510073 


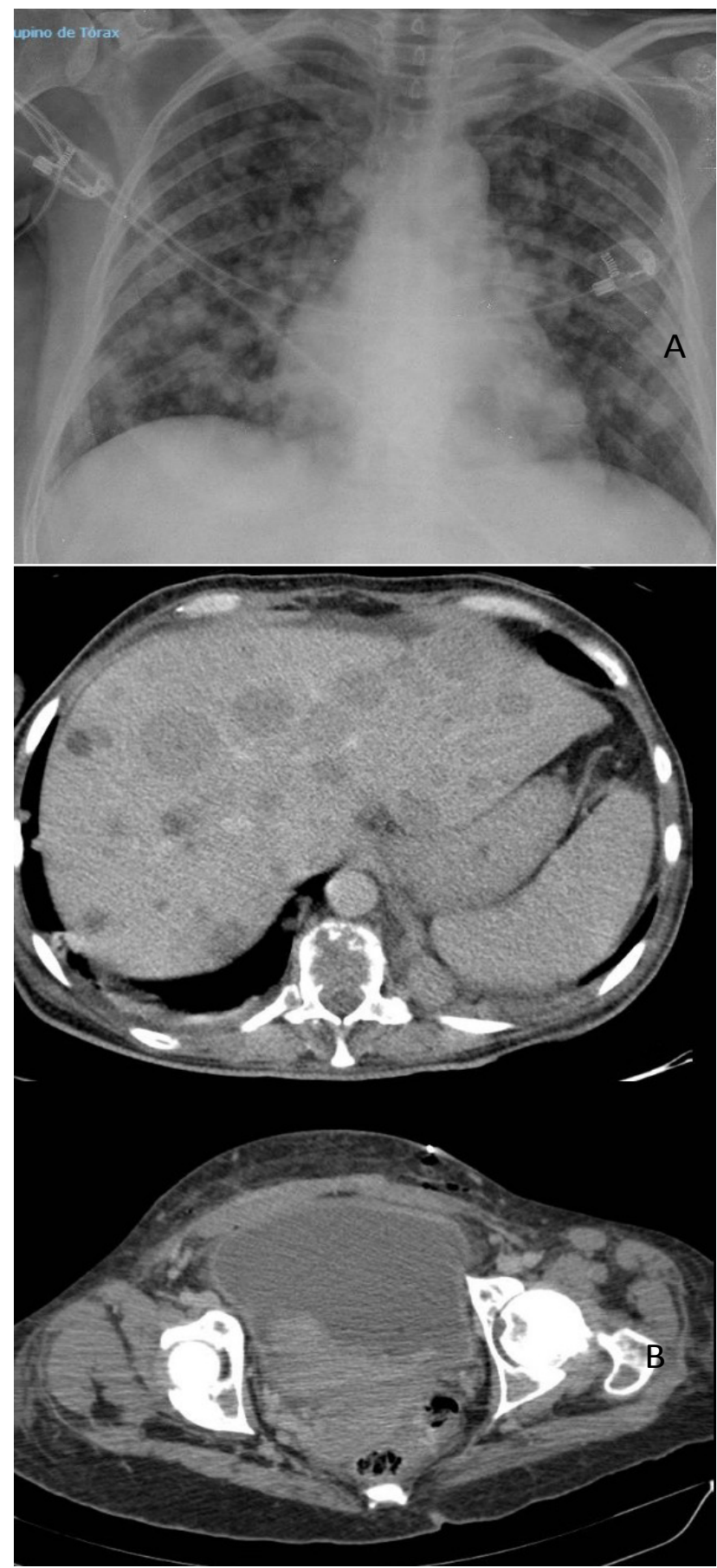

Figure 1: A) Cottony infiltrates at X-Ray; B) TC scan showing bladder lesion and metastatic lesions at liver.

opsies for etiologic diagnosis. At TUR, it was evidenced by brown excrescence bladder lesion appearance. Microscopic after study shows spindle cell tumour proliferation with areas of necrosis tested positive for S-100, Melan-A and HMB-45 (Figure 2).

After the microscopic study, the patient was studied by the dermatology department without finding any malignant skin lesions.

The BRAF study was negative. The extension study was completed with skull CT scan finding clivus lesions and other locations.

Our patient was presented in an oncology commission to determinate the best treatment option, and it

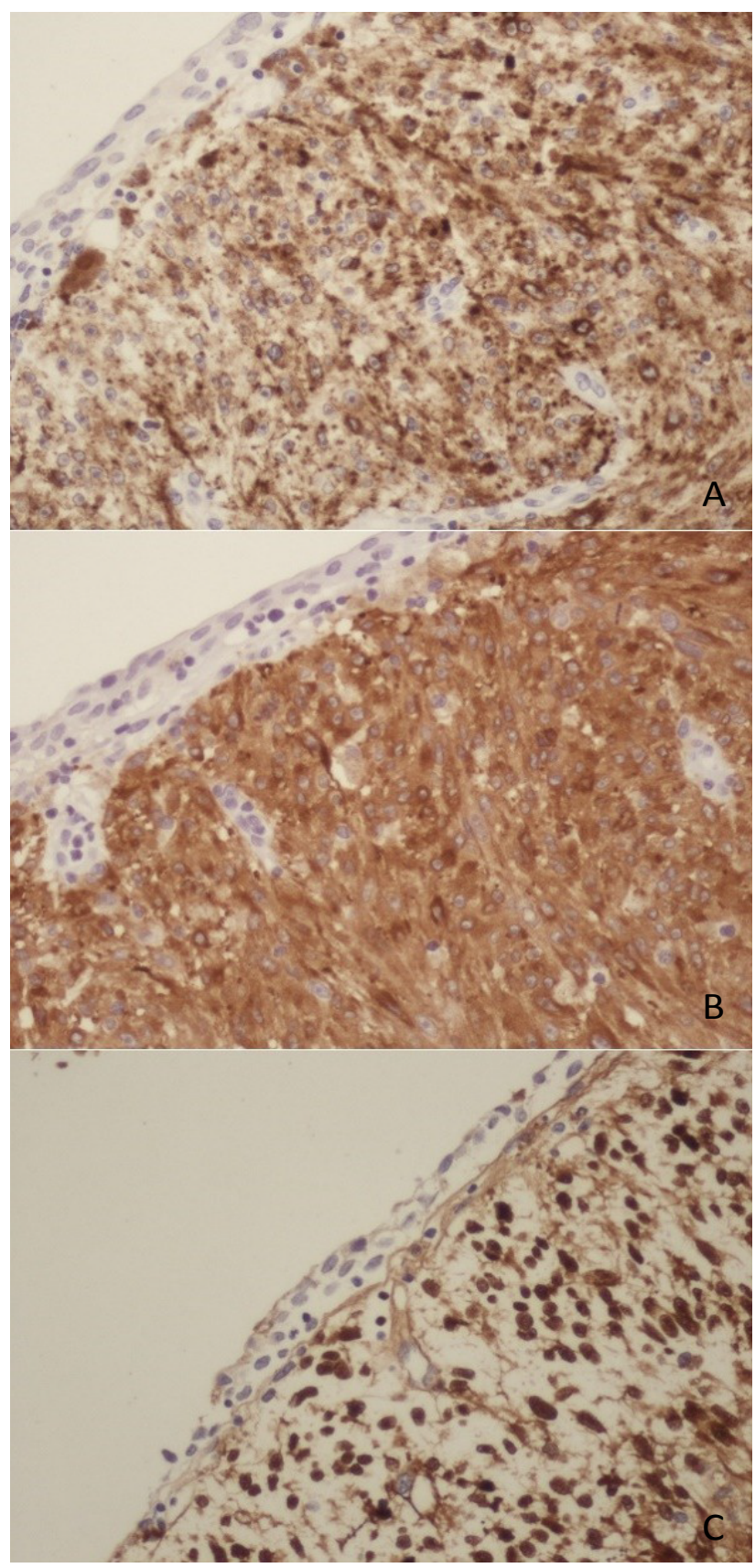

Figure 2: A) HMB45 positive; B) Melan A2 positive; C) S100 positive.

was decided to initiate chemotherapy treatment. Oncology department started the management by submitting the patient to be included in a clinical trial with Nivolumab, but it was not approved, so therapy with Temozolomide was started.

After the treatment was started, the patient underwent respiratory failure and right ventricular failure as well as the progression of the disease, requiring hospital admission with subsequent deterioration, ending the patient's death one and a half months after the diagnosis.

All decision and management of the patient were made under the express consent of the patient.

Primary bladder melanoma is an infrequent entity; the most common is the appearance of bladder melano- 
ma as a metastatic disease from another location. It has been postulated as a possible aetiology of this histology the origin of these cells at the neural crest and the presence of these in the bladder as the remains after migration of these cells $[7,8]$.

Clinically, the most common symptom and the sign of presentation is hematuria as in the rest of bladder tumours. Other symptoms include urinary tract infection, symptoms of renal colic, low urinary tract symptoms, etc. There is a case published by Lamichhane and Dhakal in which the first symptom presented by the patient was acute urinary retention [7].

For diagnosis, Ainsworth establishes in 1976 specific criteria for the correct definition of bladder melanoma. These include (1) Careful physical examination including the skin with Wood's light together with a detailed history to exclude cutaneous melanoma, (2) Exclusion of visceral melanoma following exhaustive evaluation, (3) Pattern of recurrence consistent with primary melanoma of urinary bladder and (4) Histologically proved primary atypical melanocytes $[9,10]$.

In the treatment, numerous techniques have been described in the literature, but it is crucial to evaluate metastatic workup before commencing the aggressive treatment option.

Transurethral resection, partial cystectomy, radical cystectomy, chemotherapy and radiation therapy have been used to treat bladder melanoma. It has been postulated that radical surgery for localized lesions is the reference treatment. In our case, it is a disseminated disease; surgery alone was raised as a diagnostic method to shed information about the histology of bladder injury [11-13].

Bladder melanoma prognosis is poor; to date, very little of the patients survived more than three years despite radical treatment $[9,10]$.

It is rare to find mutation on BRAF V600E in the cases of mucosal melanoma, although the literature describes the rise of the levels of mutated BRAF at urinary bladder melanoma. In our case, the study about mutated BRAF was negative, but maybe more information about melanoma should be found to conclude this [14].

Our case shows a particular scene; the patient was a pregnant woman. Although rare, cases have been described in which pregnant women presenting with neoplasms have transmitted these cell lines to their children giving rise to disease states in these, for example, a transmission from mother to offspring of a leukemic cell clone. Malignant melanoma has been shown to be the cancer with the highest maternal-fetal transmission rate $[14,15]$.

The fact that the rate of marteno-fetal transmission of malignant tumour cells is so low reveals the efficacy of the placental barrier as an immunosurveillance modulating mechanism. It is established that the loss of certain HLA can contribute to the passage of malignant cells through this barrier.

In our case, the child was born without any sign of disease, and his evolution from his birth has been according to standard parameters.

Tellez, et al. suggest that pregnancy significantly affects the results of melanoma; it has even been described that melanoma associated with pregnancy is associated with up to a $56 \%$ higher risk of mortality. This evidence is further supported by some studies that suggest that pregnancy results in reduced antitumor immunity and increased tumour lymphangiogenesis $[14,16]$.

\section{Conclusions}

Malignant melanoma of urinary bladder is a sporadic disease. Its diagnose is difficult, and its late diagnosis is frequent. Considering the diagnosis of primary bladder melanoma, it is essential to rule out other locations of more common origin. Multidisciplinary management is crucial for the correct treatment of the patient.

\section{Conflict of Interests}

The authors declare that they have no conflict of interests.

\section{Acknowledgement}

All authors share equal responsibility for its content and agree with the submission in its present format, and the material is original research.

\section{References}

1. Ainsworth $A M$, Clark WH, Mastrangelo $M$, Conger KB (1976) Primary malignant melanoma of the urinary bladder. Cancer 37: 1928-1936.

2. Bhutani N, Kajal P, Pawar D (2017) Primary malignant melanoma of the female urethra: Report of a rare neoplasm of the urinary tract. Int J Surg Case Rep 41: 319-322.

3. Chang AE, Karnell LH, Menck HR (1998) The National cancer data base report on cutaneous and noncutaneous melanoma: A summary of 84,836 cases from the past decade. The American college of surgeons commission on cancer and the American cancer society. Cancer 83: 1664-1678.

4. El Ammari JE, Ahallal Y, El Fassi MJ, Farih MH (2011) Primary malignant melanoma of the urinary bladder. Case Rep Urol 2011.

5. Isoda T, Ford AM, Tomizawa D, van Delft FW, De Castro DG, et al. (2009) Immunologically silent cancer clone transmission from mother to offspring. Proc Natl Acad Sci USA 106: $17882-17885$.

6. Tellez A, Rueda S, Conic RZ, Powers K, Galdyn I, et al. (2016) Risk factors and outcomes of cutaneous melanoma in women less than 50 years of age. J Am Acad Dermatol 74: 731-738.

7. Karabulut YY, Erdogan S, Sayar H, Ergen A, Ertoy Baydar D (2016) Primary malignant melanoma of the urinary bladder: Clinical, morphological, and molecular analysis of five cases. Melanoma Res 26: 616-624. 
8. Sayar H, Erdogan S, Adamhasan F, Gurbuz E, Inci MF (2014) Malignant melanoma of the bladder: A case report. Can Urol Assoc J 8: E54-E56.

9. Shukla A, Wingate JT, Baker KC, Brand TC (2016) Hexaminolevulinate blue-light cystoscopy in a patient with metastatic melanoma of the bladder. J Endourol Case Rep 2: 68-70.

10. Siroy AE, MacLennan GT (2011) Primary melanoma of the bladder. J Urol 185: 1096-1097.

11. Pacella M, Gallo F, Gastaldi C, Ambruosi C, Carmignani G (2006) Primary malignant melanoma of the bladder. Int $\mathrm{J}$ Urol 13: 635-637.

12. Raso A, Mascelli S, Nozza P, Biassoni R, Negri F, et al. (2010) Detection of transplacental melanoma metastasis using quantitative PCR. Diagn Mol Pathol 19: 78-82.
13. Smith MA, Fine JA, Barnhill RL, Berwick M (1998) Hormonal and reproductive influences and risk of melanoma in women. Int J Epidemiol 27: 751-757.

14. Venyo AK (2014) Melanoma of the urinary bladder: A review of the literature. Surg Res Pract 2014.

15. Alomari AK, Glusac EJ, Choi J, Hui P, Seeley EH, et al. (2015) Congenital nevi versus metastatic melanoma in a newborn to a mother with malignant melanoma - diagnosis supported by sex chromosome analysis and imaging mass spectrometry. J Cutan Pathol 42: 757-764.

16. Sutton B, Chan R, Sutton M, Boone T (2013) Primary malignant melanoma of the genitourinary tract with upper and lower tracts involvement. Case Rep Urol 2013. 